\title{
CONTINUITY OF HOMOMORPHISMS ON A BAIRE GROUP
}

\author{
ISIDORE FLEISCHER AND TIM TRAYNOR ${ }^{1}$
}

\begin{abstract}
A pointwise converging sequence of continuous homomorphisms is equicontinuous.
\end{abstract}

Banach [1, Satz 7, p. 108] proved that the pointwise limit of a sequence of continuous homomorphisms on a Baire group is continuous. This was refined by Pettis [5, Corollary 2.1, p. 297] who showed that a pointwise converging sequence of continuous homomorphisms on a Baire group is equicontinuous-which entails the continuity of the limit. His proof is dense, appealing to extraneous concepts and external previously proved results and imposing on the reader a modification of the major portion of the preceding theorem's proof. We present a direct, self-contained, and uncluttered proof of a somewhat more general result: A sequence of continuous homomorphisms which is pointwise right- (or left-) Cauchy on a nonmeager subset of a topological group is equicontinuous; whence, its limit is continuous wherever it exists.

Let $f_{n}$ be a sequence of continuous functions from a topological space $X$ to a uniform space $Y$ which is pointwise Cauchy, and let $V$ be an entourage of $Y$ closed in $Y \times Y$ : the set of $x$ at which $\left(f_{m} x, f_{n} x\right) \in V$ is closed in $X$ and therefore so is the intersection of these sets for $m \geqslant n$. These intersections increase with $n$, and the content of pointwise Cauchyness on a set $C$ is just that their union contains $C$. If $C$ is nonmeager, one of these must have nonvoid interior and thus may, if $X$ is a group, be written as a translate $U x$ of a neighborhood of the identity $e$. Taking also $Y$ to be a group uniformized by its right translates and the $f_{n}$ to be homomorphisms, this yields $f_{m} u=f_{m} u x\left(f_{n} u x\right)^{-1} f_{n} u\left(f_{m} x f_{n} x^{-1}\right)^{-1} \in V f_{n} u V^{-1}$ for $u \in U$ and $m \geqslant n$. Since $f_{n}$ is continuous at $e$, it follows that $f_{m} u \in V V V^{-1}$ for all $m \geqslant n$ on some possibly smaller $U .^{2}$ Since there are only finitely many preceding $m$, this entails the equicontinuity of the $f_{n}$ and the continuity of their limit.

Pettis postulates pointwise convergence of the sequence of homomorphisms (rather than their one-sided Cauchyness) on a nonmeager subset with the Baire property, that is, one which differs by a meager set from an open set ("almost open" in Bourbaki). The presence of such a subset entails that the group is Baire (which we do

Received by the editors November 20, 1983 and, in revised form, March 26, 1984.

1980 Mathematics Subject Classification. Primary 22A10.

Key words and phrases. Equicontinuity, Baire, topological group.

${ }^{1}$ Both authors benefited from NSERC Operating Grants.

${ }^{2}$ Thus, $f_{n} u$ converges to $e$ in $Y$ along the product filter, Fréchet in $\mathbf{N}$ by neighborhoods of $e$ in $X$; that is, $f_{n}$ converges continuously at $e$. 
not require); on the other hand, there are nonmeager subsets of the real line, which are not almost open (Bourbaki [2, IX.5, Exercise 27]).

A different generalization of Banach's theorem is offered in [3].

\section{REFERENCES}

1. S. Banach, Über metrische Gruppen, Studia Math. 3 (1931), 101-113.

2. N. Bourbaki, General topology, Hermann, Paris, and Addison-Wesley, Reading, Mass., 1966.

3. I. Fleischer and T. Traynor, Equicontinuity and uniform boundedness for homomorphisms and measures, Windsor Math. Report \#83-16.

4. J. L. Kelly and I. Namioka, Linear topological spaces, Van Nostrand, Princeton, N. J., 1963.

5. B. J. Pettis, On continuity and openness of homomorphisms in topological groups, Ann. of Math. (2) 52 (1950), 293-308.

C. R. M. A., Universite de Montreal, Montreal H3C 3J7, Quebec, Canada

Department of Mathematics, University of Windsor, Windsor N9B 3P4, Ontario, Canada 